\section{Estrategias para la disminución de la mortalidad por neumonía}

\section{Strategies for decreasing pneumonia mortality}

\section{Señor Editor:}

Hemos leído con interés el estudio de M. L. Rioseco et $\mathrm{al}^{1}$ que pone de manifiesto la elevada mortalidad de la neumonía bacteriémica por neumococo. Nos parece un tema de enorme interés por la repercusión en salud que tiene esta enfermedad y nos gustaría hacer algunos comentarios a raíz del excelente artículo publicado.

En primer lugar, queremos compartir los resultados del análisis de las neumonías incluidas en el registro del Grupo de Infecciones de la Sociedad Española de Medicina de Emergencias (INFURG-SEMES). Se trata de un registro multipropósito que recoge todos los pacientes en los que se aisló algún microorganismo en una muestra tomada en el Servicio de Urgencias durante el año 2017 en 57 hospitales españoles. Se incluyeron 5682 pacientes, de los cuales 437 presentaban neumonía. La edad media de los pacientes incluidos fue de 74,3 (DE 16,1) años, siendo $279(63,8)$ varones. Presentaban un índice de Charlson $\geq 3$ un total de 201 $(46,0 \%)$ pacientes. Desde el punto de vista de la estratificación del riesgo, $44(10,1 \%)$ tenían un qSOFA $\geq 2$ y $84(19,2 \%)$ una puntuación en la escala de NEWS $\geq 7$, es decir un riesgo clínico alto de malos resultados. El neumoco, aislado en $79(18,1 \%)$ de los pacientes y el Haemophilus influenzae en 46 (10,5\%) fueron los patógenos más frecuentemente identificados. La mortalidad a 30 días se produjo en $86(19,7 \%)$ de los pacientes. El tratamiento inapropiado ocurrió en 75 (17,2\%) sujetos. Estos resultados están en consonancia con lo comunicado por Rioseco et al $^{1}$ en cuanto a la elevada mortalidad de la neumonía.

Además, queremos destacar la elevada frecuencia de tratamiento inapropiado. Debemos reseñar que aunque las resistencias a neumococo han ido disminuyendo tras la introducción de la vacuna conjugada 7 valente en el calendario vacunal infantil en España, y los puntos de corte de la concentración mínima inhibitoria se ha modificado al alza, es aconsejable administrar dosis altas de betalactámicos para alcanzar niveles séricos adecuados y poder actuar frente al neumococo con nivel intermedio de resistencia ${ }^{2}$.

La neumonía es la primera causa de sepsis y una de las principales causas de muerte en el mundo ${ }^{3}$. Debido a su alta mortalidad es imprescindible realizar una adecuada estratificación del riesgo de muerte en los pacientes que atendemos por neumonía, lo que nos permitirá adecuar el tratamiento antibiótico empírico inicial y tomar las primeras decisiones en lo referido a la ubicación, tanto desde el punto de vista de la decisión de ingreso o alta como del lugar de ingreso.
Estas decisiones iniciales son fundamentales de cara a reducir la mortalidad de los pacientes que atendemos por infección $n^{4,5}$.

De cara a la estratificación del riego, en la práctica clínica habitual utilizamos escalas clínicas y biomarcadores de respuesta inflamatoria (BMRI). En lo referente a las escalas clínicas, las más utilizadas son el CURB-65 y el PSI (Pneumonia Severity Index). Sin embargo, es conocido que estas escalas tienen importantes limitaciones. En el caso del CURB-65 presenta la limitación de no incluir en la valoración la hipoxemia y la situación funcional. Tampoco es útil para valorar la necesidad de ingreso en Cuidados Intensivos. El PSI identifica bien el bajo riesgo de mortalidad y nos ayuda a decidir "el alta", pero puede infraestimar la gravedad, sobre todo en jóvenes o en pacientes con enfermedad pulmonar obstructiva crónica ${ }^{6}$.

Los BMRI pueden tener limitaciones en cuanto a sensibilidad y especificidad, además del problema de su coste y la necesidad de disponer de la tecnología necesaria para su realización, aspecto que dependerá del nivel asistencial en el que nos encontremos. La ventaja es que son medidas objetivas y cuantitativas que bien interpretadas pueden facilitar la toma de decisiones.

Otro aspecto fundamental a considerar es que la incidencia de neumonía se incrementa con la edad. El paciente anciano presenta con más frecuencia manifestaciones clínicas atípicas y una respuesta inflamatoria más pobre, lo que dificulta la estratificación adecuada de la gravedad. Estudios previos del Grupo INFURG-SE$\mathrm{MES}^{7,8} \mathrm{han}$ mostrado que los biomarcadores pueden ser de gran utilidad en la estratificación del riesgo de los pacientes ancianos infectados, destacando en este sentido la procalcitonina, que logra un área bajo la curva $(\mathrm{ABC})$ para predecir mortalidad a corto plazo de $0,734[(0,581$ $0,888) ; p=0,006]$, la determinación de la región medial de la proadrenomodulina (MRproADM), que presenta un $\mathrm{ABC}$ de 0,886 [ $(0,775-0,997) ; \mathrm{p}<0,001]$, y el lactato con un $\mathrm{ABC}$ de 0,823 [(0,710-0,936); $\mathrm{p}<0,001]$. No obstante, el modelo combinado de MRproADM con qSOFA $\geq 2$ ofrece el mejor rendimiento pronóstico, con una sensibilidad del $69 \%$ y una especificidad de $97 \%$ [ABC 0,878 (IC 95\% 0,749-1; p < 0,001)].

Por otra parte, la vacunación en el anciano ha demostrado reducir los casos de muerte, así como las complicaciones asociadas a pesar de la menor respuesta consecuencia de la inmunosenescencia, por lo que todo paciente anciano debería estar vacunado. La vacuna polisacárida (VNP23) frente a neumococo, que se emplea desde hace décadas, aunque incluye el mayor número de serotipos no genera memoria inmunitaria y provoca en la población anciana una pobre respuesta inmunitaria resultando clínicamente ineficaz. En sujetos inmunocompetentes con patologías de basese recomienda la vacuna conjugada (VNC13), que genera memoria inmunitaria y una respuesta inmune más 
potente que la vacuna polisacárida, lo que redunda en un claro beneficio predecible .

Para disminuir la importante mortalidad de esta patología, desde INFURG-SEMES hemos diseñado distintos protocolos y publicaciones y abogamos por la implantación de una guía de práctica clínica (GPC), ya que en general éstas condicionan una mejora significativa de todo el proceso asistencial tras su implantación ${ }^{10}$. Las GPC se pueden definir como una serie de recomendaciones sistemáticas, basadas en la mejor evidencia de la literatura médica, realizadas con el objetivo de asistir al médico en la toma de decisiones.

En conclusión, en nuestra opinión, la vacunación del paciente anciano frente al neumococo y la utilización de GPC para la estratificación del riesgo y el manejo terapéutico inicial son los pilares fundamentales que pueden contribuir a disminuir la mortalidad de esta infección.

Juan González del Castillo ${ }^{1,2}$, Beatriz Valle Borrego ${ }^{3}$, Ferrán Llopis Roca ${ }^{4}$, María José Núñez-Orantos ${ }^{4,5}$

${ }^{1}$ Servicio de Urgencias, Hospital Clínico San Carlos, Madrid, España.

${ }^{2}$ Instituto de Investigación Sanitaria del Hospital San Carlos, Madrid, España.

${ }^{3}$ Servicio de Urgencias, Hospital Universitario Severo Ochoa, Madrid, España.

${ }^{4}$ Servicio de Urgencias. Hospital Universitario de Bellvitge, Barcelona, España.

${ }^{5}$ Servicio de Medicina Interna, Hospital Clínico San Carlos, Madrid, España.

\section{Referencias}

1. Rioseco ZML, Riquelme OR, Riquelme OM, Inzunza PC, Riquelme DJ, Sanhueza RA. Neumonía neumocócica bacteriémica en adultos en hospital regional de Chile. Rev Med Chile 2018; 146: 839-45.

2. OltraHostalet F, Núñez-Núñez M, Portillo Cano MM, Navarro Bustos C, Rodríguez-Baño J, Retamar Gentil P. Análisis de la calidad de uso de antimicrobianos en el servicio de urgencias de un hospital de tercer nivel. Emergencias 2018; 30: 297-302.

3. Torres Bonafonte OH, Gil Olivas E, Pérez Macho E, Pacho Pacho C, Mateo Roca M, CasademontPou J, et al. Predictores de patógenos resistentes en las neumonías procedentes de la comunidad: ¿es útil en urgencias el concepto de neumonía asociada a cuidados sanitarios? Emergencias 2017; 29: 30612.

4. García-Villalba E, Cano-Sánchez A, Alcaraz-García A, Cinesi-Gómez C, Piñera-Salmerón P, Marín I, et al. Nomograma para predecir mal pronóstico en pacientes procedentes de urgencias con sepsis y bajo riesgo de daño orgánico evaluado mediante SOFA. Emergencias 2017; 29: 81-6.

5. García-Lamberechts EJ, Martín-Sánchez FJ, Julián Jiménez A, Llopis F, Martínez Ortiz de Zárate M, Arranz Nieto MJ, et al. Modelo de riesgo a 30 días en los pacientes ancianos con infección y síndrome de respuesta inflamatoria sistémica atendidos en los servicios de urgencias. Emergencias 2018; 30: 241-6.

6. Julián-Jiménez A, González Del Castillo J, Candel FJ. Usefulness and prognostic value of biomarkers in patients with community-acquired pneumonia in the emergency department. Med Clin (Barc) 2017; 148: 501-10.

7. Yáñez Palma MC, Salido-Mota M, Guardiola JM, Debán Fernández M, López Izquierdo R, González Del Castillo J. Utilidad de un panel de biomarcadores para estratificar el riesgo de muerte a 30 días en el paciente anciano atendido por infección aguda en urgencias. Emergencias 2018; 30: 354-5.

8. Julián-Jiménez A, Yáñez MC, González del Castillo J, Salido-Mota M, Mora-Ordoñez B, Arranz-Nieto MJ, et al; en representación del grupo INFURG-SEMES. Prognostic power of biomarkers for short-term mortality in the elderly patients seen in Emergency Departments due to infections.EnfermInfeccMicrobiolClin. 2017. pii: S0213005X(17)30381-6. doi: 10.1016/j.eimc.2017.11.017. [Epubahead of print].

9. Picazo J, González-Romo F, García-Rojas A, Pérez-Trallero E, Gil Gregorio P, de la Cámara R, et al. Consensus document on pneumococcal vaccination in adults with risk underlying clinical conditions. Rev Esp Quimioter 2013; 26: 232-52.

10. Grupo de Infecciones de la Sociedad Española de Medicina de Urgencias y Emergencias (INFURG-SEMES). Consultado el 14 de noviembre de 2018, de http://www.infurg-semes.org

Correspondencia:

Juan González del Castillo

Servicio de Urgencias. Hospital Clínico San Carlos.

Calle Profesor Martín-Lagos s/n, 28040 Madrid, España.

jgonzalezcast@gmail.com 